\title{
A model calculation of double parton distribution functions of the pion
}

\author{
Matteo Rinaldi ${ }^{1,2, a}$, Sergio Scopetta ${ }^{3,4}$, Marco Traini $^{5,6}$, Vicente Vento $^{1,2}$ \\ ${ }^{1}$ Departament de Fisica Teòrica, Universitat de València, Valencia, Spain \\ ${ }^{2}$ Institut de Fisica Corpuscular, Consejo Superior de Investigaciones Científicas, 46100 Burjassot, València, Spain \\ ${ }^{3}$ Dipartimento di Fisica e Geologia, Università degli Studi di Perugia, Perugia, Italy \\ ${ }^{4}$ Istituto Nazionale di Fisica Nucleare, Sezione di Perugia, Perugia Via A. Pascoli, 06123 Perugia, Italy \\ ${ }^{5}$ INFN - TIFPA, Via Sommarive 14, I-38123 Trento-Povo, Italy \\ ${ }^{6}$ Dipartimento di Fisica, Universit Ã degli Studi di Trento, Via Sommarive 14, I-38123 Trento-Povo, Italy
}

Received: 2 July 2018 / Accepted: 15 September 2018 / Published online: 26 September 2018

(C) The Author(s) 2018

\begin{abstract}
Two-parton correlations in the pion are investigated in terms of double parton distribution functions. A Poincaré covariant light-front framework has been adopted. As non perturbative input, the pion wave function obtained within the so-called soft-wall AdS/QCD model has been used. Results show how novel dynamical information on the structure of the pion, not accessible through one-body quantities, are encoded in double parton distribution functions.
\end{abstract}

\section{Introduction}

Double parton scattering (DPS), the simplest form of multiple parton interaction (MPI), has been observed at the LHC (see, e.g., Ref. [1]). The DPS cross section can be written in terms of double parton distribution functions (dPDFs) [2-4], which represent the number density of two partons located at a given transverse separation in coordinate space and with given longitudinal momentum fractions. This is an information complementary to the tomography accessed through electromagnetic probes in terms of generalized parton distributions (GPDs) [5,6]. If measured, dPDFs would therefore represent a novel tool to access the three-dimensional hadron structure [7]. However, since dPDFs describe soft Physics, they are non perturbative objects and have not been evaluated in QCD. It is therefore useful to estimate them at low momentum scales $\left(\sim \Lambda_{Q C D}\right)$, for example using quark models as it has been proposed in Refs. [8-13]. In order to match theoretical predictions with future experimental analyses, the results of these calculations are then evolved using perturbative QCD to reach the high momentum scale of the data $[14,15]$.

\footnotetext{
a e-mail: mrinaldi@ific.uv.es
}

In a previous work, use has been made of the AdS/QCD framework to study dPDFs in proton-proton collisions [13]. The AdS/QCD approach establishes a correspondence between conformal field theories and gravitation in an antide-Sitter space [16-18]. The so-called bottom-up approach implements important features of QCD, generating a theory in which conformal symmetry is restored asymptotically [19-22]. This approach has been successfully applied to the description of the spectrum of hadrons, of their form factors (ffs) and parton distributions (PDFs) [23-28]. In particular the structure of the pion is an interesting subject which has attracted much attention from the point of view of AdS/QCD [23,29,30]. In this scenario we proceed here to generalize the formalism developed for nucleon dPDFs to mesons and apply it using pion wave functions defined via the AdS/QCD correspondence. One should notice that, for nucleons, DPS data should become available and the extraction of dPDFs, although difficult, could be obtained from LHC data in the future. Measurements of this kind for mesons appear much more challenging. Nonetheless, lattice data for moments of dPDFs of mesons can become available, while the same appears much more difficult for the nucleon. As a matter of facts, our analysis has been partially motivated by a first estimate of moments of quantities related to pion dPDFs in the lattice, recently reported [31]. The possibility to access the specific non perturbative information from lattice is indeed rather interesting.

In Sect. 2 we describe the spin-independent meson dPDF in terms of the light-front (LF) wave function (w.f.), and introduce an approximation which relates dPDFs to GPDs and ffs. Furthermore we introduce a quantity relevant to DPS phenomenology, the effective cross section $\sigma_{e f f}$, in terms of dPDFs and PDFs. In Sect. 3 AdS/QCD model calculations 
of dPDFs are summarized and their properties analyzed. In Sect. 4 the evolution of the dPDFs to high momentum scale is calculated and its implications discussed. Conclusions are collected in Sect. 5.

\section{Double PDF and the meson light-front wave function}

Due to the explorative character of this investigation, we discuss the most natural $\mathrm{dPDF}$, the unpolarized one given in terms of the Dirac structure $\gamma^{\mu}$. In this section we describe how to express it in terms of the light-front (LF) meson wave function. The formalism we use has been also presented in Ref. [32], where dPDFs have been studied for a dressed quark target treated as a two body system. Formally, the spin-independent dPDF is defined by means of the lightcone correlator [3],

$$
\begin{aligned}
F_{q_{1} q_{2}}\left(x_{1}, x_{2}, \mathbf{k}_{\perp}\right) & \\
= & \frac{P^{+}}{4} \int d^{2} \mathbf{y}_{\perp} e^{-i \mathbf{y}_{\perp} \cdot \mathbf{k}_{\perp}} \int d y^{-} \int d z_{1}^{-} d z_{2}^{-} \\
& \times \frac{e^{-i x_{1} P^{+} z_{1}^{-}-i x_{2} P^{+} z_{2}^{-}}}{(2 \pi)^{2}} \\
& \times\left.\left\langle A, \mathbf{0}\left|\mathcal{O}_{q_{1}}\left(0, z_{1}\right) \mathcal{O}_{q_{2}}\left(y, z_{2}\right)\right| A, \mathbf{0}\right\rangle\right|_{y^{+}=z_{1}^{+}=z_{2}^{+}=0} ^{\mathbf{z}_{1}=\mathbf{z}_{\perp}=0}
\end{aligned}
$$

where for generic 4-vectors $y$ and $z$, the operator $\mathcal{O}_{q}(y, z)$ for the quark of flavor $q$ reads:

$\mathcal{O}_{q}(y, z)=\bar{q}\left(y-\frac{1}{2} z\right) \gamma^{+} q\left(y+\frac{1}{2} z\right)$,

and $q(z)$ is the LF quark field operator. In order to find a suitable expression of the dPDF, we make use of the LF wave function representation approach $[33,34]$. In particular, the pion minimal $(q \bar{q})$ Fock-state configuration is written as

$$
\begin{aligned}
\left|A, \mathbf{P}_{\perp}\right\rangle= & \sum_{h, \bar{h}} \int \frac{d x_{1} d x_{2}}{\sqrt{x_{1} x_{2}}} \frac{d^{2} \mathbf{k}_{\mathbf{1}} \perp d^{2} \mathbf{k}_{\mathbf{2} \perp}}{2(2 \pi)^{3}} \delta^{(2)}\left(\mathbf{k}_{\mathbf{1} \perp}+\mathbf{k}_{\mathbf{2} \perp}\right) \\
& \times\left|x_{1}, \mathbf{k}_{\mathbf{1} \perp}+x_{1} \mathbf{P}_{\perp}, h\right\rangle\left|x_{2}, \mathbf{k}_{\mathbf{2} \perp}+x_{2} \mathbf{P}_{\perp}, \bar{h}\right\rangle \\
& \times \delta\left(1-x_{1}-x_{2}\right) \psi_{h, \bar{h}}^{\pi}\left(x_{1}, x_{2}, \mathbf{k}_{\mathbf{1} \perp}, \mathbf{k}_{\mathbf{2} \perp}\right) .
\end{aligned}
$$

Here, $h$ and $\bar{h}$ represent parton helicities, $x_{i}=k_{i}^{+} / P^{+}$and $\mathbf{k}_{\mathbf{i} \perp}$ the quark longitudinal momentum fraction and its transverse momentum, respectively, $P^{\mu}$ the meson 4-momentum. The light cone components are defined by $l^{ \pm}=l^{0} \pm l^{3}$. In Eq. (3), $\psi_{h, \bar{h}}^{\pi}\left(x_{1}, x_{2}, \mathbf{k}_{\mathbf{1} \perp}, \mathbf{k}_{\mathbf{2}} \perp\right)$ is the LF meson wave-function, whose normalization is chosen as

$$
\begin{aligned}
1= & \frac{1}{2} \sum_{h, \bar{h}} \int d x_{1} d x_{2} \frac{d^{2} \mathbf{k}_{\mathbf{1}} \perp d^{2} \mathbf{k}_{\mathbf{2}} \perp}{16 \pi^{3}} \delta\left(1-x_{1}-x_{2}\right) \\
& \times \delta^{(2)}\left(\mathbf{k}_{\mathbf{1} \perp}+\mathbf{k}_{\mathbf{2} \perp}\right)\left|\psi_{h, \bar{h}}^{\pi}\left(x_{1}, x_{2}, \mathbf{k}_{\mathbf{1} \perp}, \mathbf{k}_{\mathbf{2}} \perp\right)\right|^{2} .
\end{aligned}
$$

The w.f. $\psi_{h, \bar{h}}^{\pi}\left(x_{1}, x_{2}, \mathbf{k}_{1} \perp, \mathbf{k}_{\mathbf{2}} \perp\right)$ determines the structure of the state and is not known.

However one can obtain the dPDF by using a standard procedure (see e.g. Ref. [10] for the proton) which makes use of the quark-antiquark field operator [23], the definition of the meson state Eq. (3), of Eq. (1) and the anticommutation relations between creation-annihilation operators (see Ref. [23] for details). The result for the pion dPDF is

$$
\begin{aligned}
F_{q_{1} \bar{q}_{2}}\left(x_{1}, x_{2}, \mathbf{k}_{\perp}\right) \\
=\frac{1}{2} \sum_{h, \bar{h}} \int \frac{d^{2} \mathbf{k}_{\mathbf{1} \perp}}{2(2 \pi)^{3}} \psi_{h, \bar{h}}^{\pi}\left(x_{1}, x_{2}, \mathbf{k}_{\mathbf{1} \perp},-\mathbf{k}_{\mathbf{1} \perp}\right) \\
\quad \times \psi_{h, \bar{h}}^{* \pi}\left(x_{1}, x_{2}, \mathbf{k}_{\mathbf{1} \perp}+\mathbf{k}_{\perp},-\mathbf{k}_{\mathbf{1} \perp}-\mathbf{k}_{\perp}\right) \\
\quad \times \delta\left(1-x_{1}-x_{2}\right) \\
=f_{2}^{\pi}\left(x_{1}, \mathbf{k}_{\perp}\right) \delta\left(1-x_{1}-x_{2}\right) .
\end{aligned}
$$

In the above expression, $q_{1}$ and $\bar{q}_{2}$ are the flavors present in the considered pion type, $\pi$. In general, the object of physical interest here is $f_{2}^{\pi}\left(x_{1}, \mathbf{k}_{\perp}\right)$, obtained as integral over $x_{2}$ of $F_{q_{1} \bar{q}_{2}}\left(x_{1}, x_{2}, \mathbf{k}_{\perp}\right)$ and given by

$$
\begin{aligned}
& f_{2}^{\pi}\left(x, \mathbf{k}_{\perp}\right) \\
& \quad=\frac{1}{2} \sum_{h, \bar{h}} \int \frac{d^{2} \mathbf{k}_{\mathbf{1} \perp}}{2(2 \pi)^{3}} \psi_{h, \bar{h}}\left(x, \mathbf{k}_{\mathbf{1} \perp}\right) \psi_{h, \bar{h}}^{*}\left(x, \mathbf{k}_{\mathbf{1} \perp}+\mathbf{k}_{\perp}\right) .
\end{aligned}
$$

Notice that for $\mathbf{k}_{\perp}=0$, the usual LF PDF expression is recovered [30]. Here and in the following, we use the subscript " 2 " in order to distinguish the above quantity from the PDF, denoted $f_{1}(x)$. We will calculate the quantity $f_{2}^{\pi}\left(x, \mathbf{k}_{\perp}\right)$ encoding the relevant dynamical information. Since the LF meson wave function is evaluated under the conditions $x_{2}=1-x_{1}$ and $\mathbf{k}_{\mathbf{2} \perp}=-\mathbf{k}_{\mathbf{1} \perp}$, due to momentum conservation, for simplicity, we use the notation

$\psi_{h, \bar{h}}\left(x_{1}, \mathbf{k}_{\mathbf{1} \perp}\right)=\psi_{h, \bar{h}}^{\pi}\left(x_{1}, 1-x_{1}, \mathbf{k}_{\mathbf{1} \perp},-\mathbf{k}_{\mathbf{1} \perp}\right)$.

In the following, in order to distinguish the dPDF $F_{q_{1} \bar{q}_{2}}\left(x_{1}\right.$, $\left.x_{2}, \mathbf{k}_{\perp}\right)$ from $f_{2}^{\pi}\left(x, \mathbf{k}_{\perp}\right)$, we will call the latter "integrated dPDF". We are mainly interested in non perturbative aspects of the dPDFs, so that, in order to emphasize the role of correlations between $x$ and $\mathbf{k}_{\perp}$, in the next sections the following ratio will be calculated:

$r_{k}\left(x, k_{\perp}\right)=\frac{F_{q_{1} \bar{q}_{2}}\left(x, x_{2}, k_{\perp}\right)}{F_{q_{1} \bar{q}_{2}}\left(0.4, x_{2}, k_{\perp}\right)}=\frac{f_{2}^{\pi}\left(x, k_{\perp}\right)}{f_{2}^{\pi}\left(0.4, k_{\perp}\right)} ;$

in fact, if a factorized ansatz, e.g. $f_{2}^{\pi}\left(x, k_{\perp}\right) \sim f_{2, x}(x) f_{2, k_{\perp}}$ $\left(k_{\perp}\right)$, is used, $r_{k}\left(x, k_{\perp}\right)$ does not depend on $k_{\perp}[9,10,35]$. The factorization ansatz is often used in experimental analyses for the proton target. 
In closing this section, we note that the dPDFs and the integrated dPDF depend on two momentum scales, which we have not shown, corresponding to the mass of the states produced in the two parton-parton scattering in the DPS process. These scales will be the low energy scales in the evolution process. Model results are adscribed to a low momentum scale, where the pion is dominated by the $\bar{q} q$ Fock state, the so called hadronic scale $\mu_{0}^{2}$. We take both scales at the hadronic scale.

\subsection{An approximation in terms of one body quantities}

An ansatz commonly used to describe the unknown dPDFs makes use of ffs and GPDs (in the case of the proton some experimental knowledge is available). Following the strategy of Refs. [3,36,37], we consider the correlator (1) and insert a complete set of states assuming that the pion is dominant. The formal expression for this approximated quantity, $F_{q_{1} \bar{q}_{2}}^{A}\left(x_{1}, x_{2}, \mathbf{k}_{\perp}\right)$, is:

$$
\begin{aligned}
& F_{q_{1} \bar{q}_{2}}^{A}\left(x_{1}, x_{2}, \mathbf{k}_{\perp}\right)=\frac{P^{+}}{4} \int d^{2} \mathbf{y}_{\perp} e^{-i \mathbf{y}_{\perp} \cdot \mathbf{k}_{\perp}} \int d y^{-} \\
& \quad \times \int d z_{1}^{-} d z_{2}^{-} \int \frac{d P^{\prime+} d^{2} \mathbf{P}_{\perp}^{\prime}}{2(2 \pi)^{3} P^{\prime+}} \frac{e^{-i x_{1} P^{+} z_{1}^{-}-i x_{2} P^{+} z_{2}^{-}}}{(2 \pi)^{2}} \\
& \times\left\langle A, \mathbf{0}\left|\mathcal{O}_{q_{1}}\left(0, z_{1}\right)\right| A^{\prime}, \mathbf{P}_{\perp}^{\prime}\right\rangle\left\langle A^{\prime}, \mathbf{P}_{\perp}^{\prime}\left|\mathcal{O}_{\bar{q}_{2}}\left(y, z_{2}\right)\right| A, \mathbf{0}\right\rangle \\
& \quad \times\left.\right|_{y^{+}=z_{1}^{+}=z_{2}^{+}=0} ^{\mathbf{z}_{1}=\mathbf{z}_{2}=0}
\end{aligned}
$$

In this scenario, the approximation relies on the assumption $F_{q_{1} \bar{q}_{2}}\left(x_{1}, x_{2}, \mathbf{k}_{\perp}\right) \sim F_{q_{1} \bar{q}_{2}}^{A}\left(x_{1}, x_{2}, \mathbf{k}_{\perp}\right)$. At this point, using again the strategy already discussed in the previous section, we find:

$F_{q_{1} \bar{q}_{2}}^{A}\left(x_{1}, x_{2}, \mathbf{k}_{\perp}\right)=H_{q_{1}}\left(x_{1}, \mathbf{k}_{\perp}\right) H_{\bar{q}_{2}}\left(1-x_{2},-\mathbf{k}_{\perp}\right)$,

where $H_{q}\left(x, \mathbf{k}_{\perp}\right)=H_{q}\left(x, \xi=0, \mathbf{k}_{\perp}\right)$, is the pion GPD at zero skewness. The integral over $x_{2}$ of Eqs. (5) and (11) leads approximately to

$$
\begin{aligned}
f_{2}^{\pi}\left(x, \mathbf{k}_{\perp}\right) \sim f_{2, A}^{\pi}\left(x, \mathbf{k}_{\perp}\right) & =\int_{0}^{1} d x_{2} F_{q_{1} \bar{q}_{2}}^{A}\left(x, x_{2}, \mathbf{k}_{\perp}\right) \\
& =H^{\pi}\left(x, \mathbf{k}_{\perp}\right) F^{\pi}\left(\mathbf{k}_{\perp}\right),
\end{aligned}
$$

where $F^{\pi}\left(\mathbf{k}_{\perp}\right)$ is the standard pion e.m. form factor. The difference between $f_{2}^{\pi}\left(x, \mathbf{k}_{\perp}\right)$ and $f_{2, A}^{\pi}\left(x, \mathbf{k}_{\perp}\right)$ addresses the presence of unknown parton correlations that can not be studied by means of one-body distributions. This fact is evident in Eq. (11) where one sees directly that the approximation (10) amounts to neglect possible interesting correlations in longitudinal variables $x_{1}, x_{2}$. In order to expose the relevance of such effects, the relation (12) will be discussed in the next section.
The GPD for the pion [26] might be written also in terms of the LF wave function [38,39],

$$
\begin{aligned}
& H^{\pi}\left(x, \xi=0, \Delta_{\perp}^{2}\right)=\frac{1}{2} \sum_{h, \bar{h}} \int \frac{d^{2} \mathbf{k}_{\mathbf{1}} \perp}{16 \pi^{3}} \\
& \quad \times \psi_{h, \bar{h}}\left(x, \mathbf{k}_{\mathbf{1} \perp}\right) \psi_{h, \bar{h}}^{*}\left(x, \mathbf{k}_{\mathbf{1} \perp}+(1-x) \Delta_{\perp}\right),
\end{aligned}
$$

an expression well suited for model calculations which will be used in the next section in order to test the validity of the approximation Eq. (12), addressing possible new insights on the integrated dPDF. One should realize that the violation of Eq. (12) implies that $F_{q_{1} \bar{q}_{2}}\left(x_{1}, x_{2}, \mathbf{k}_{\perp}\right)$ cannot be factorized in terms of functions which can be interpreted as GPDs.

\subsection{The effective cross section}

A relevant observable for DPS proton studies is the so called effective cross section, $\sigma_{e f f}$, see e.g. Ref. [40]. It is defined as the ratio of the product of two single parton scattering process cross sections to the DPS with the same final states. It is extracted from data using model assumptions, and it can be expressed in terms of PDFs and dPDFs [11]. For protonproton collisions, this quantity has been also studied within the AdS/QCD soft-wall model [13]. In Refs. [11,13,41] it has been shown how a dependence of $\sigma_{e f f}$ on the longitudinal momentum fractions of the acting partons reflects the presence of non trivial double parton correlations. In the present study we calculate $\sigma_{e f f}$ for a meson target in order to make predictions.

Let us consider for an illustrative purpose only, the effective cross section for a DPS process involving, for example, the collision between two pions of the same charge. In general, this quantity depends on four variables $x_{1}, x_{2}$ and $x_{1}^{\prime}, x_{2}^{\prime}$, i.e. the longitudinal momentum fractions of the partons involved in the process. Nevertheless, in the zero rapidity region, i.e. $x_{1}=x_{1}^{\prime}$ and $x_{2}=x_{2}^{\prime}, \sigma_{e f f}$ reads:

$$
\sigma_{e f f}=\frac{\sum_{q_{1} q_{2} q_{3} q_{4}} C_{q_{1} q_{3}} C_{q_{2} q_{4}} f_{1}^{q_{1}}\left(x_{1}\right) f_{1}^{q_{2}}\left(x_{2}\right) f_{1}^{q_{3}}\left(x_{1}\right) f_{1}^{q_{4}}\left(x_{2}\right)}{\sum_{q_{1} q_{2} q_{3} q_{4}} C_{q_{1} q_{3}} C_{q_{2} q_{4}} \int \frac{d^{2} \mathbf{k}_{\perp}}{(2 \pi)^{2}} F_{q_{1} q_{2}}\left(x_{1}, x_{2}, \mathbf{k}_{\perp}\right) F_{q_{3} q_{4}}\left(x_{1}, x_{2},-\mathbf{k}_{\perp}\right)},
$$

where $f_{1}^{q}(x)$ is the single PDF of the flavor $q$ in the pion $\pi$, indexes in sums run over all active partons in a given process and $C_{i j}$ is a colour factor, i.e. $C_{g g}: C_{q g}: C_{q q}=1:(4 / 9)$ : $(4 / 9)^{2}$, see Ref. [11]. Furthermore, one can define an average value as follows:

$\bar{\sigma}_{e f f}=\frac{1}{\int \frac{d^{2} \mathbf{k}_{\perp}}{(2 \pi)^{2}} F_{2}^{\pi}\left(\mathbf{k}_{\perp}\right) F_{2}^{\pi}\left(-\mathbf{k}_{\perp}\right)}$, 


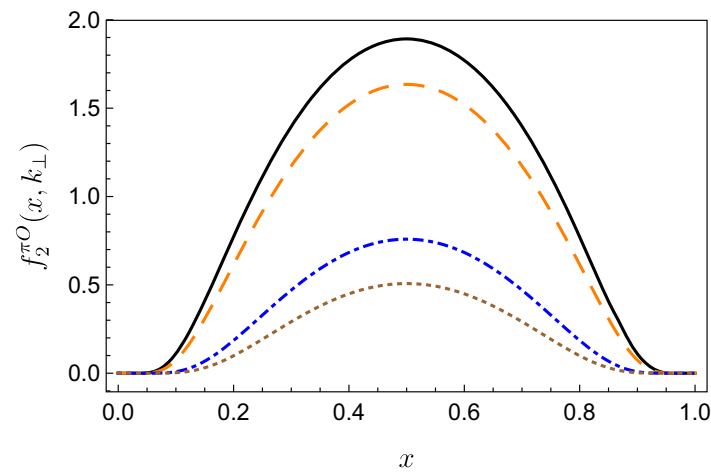

Fig. 1 Left panel: integrated dPDF of the pion within the AdS/QCD model of Ref. [23] (cfr Eq. (17)) at different values of $k_{\perp}$. Full line $k_{\perp}=0 \mathrm{GeV}$, dashed line $k_{\perp}=0.2 \mathrm{GeV}$, dot-dashed line $k_{\perp}=0.5$

where the effective form factor

$F_{2}^{\pi}\left(\mathbf{k}_{\perp}\right)=\int_{0}^{1} d x_{1} \int_{0}^{1-x_{1}} d x_{2} F_{q_{1} q_{2}}\left(x_{1}, x_{2}, \mathbf{k}_{\perp}\right)$

has been introduced (see Refs. [7,11]). Eq. (15) assumes factorization between the $x$ and $k_{\perp}$ in the dPDF. The latter quantity, Eq. (15), is the one usually studied in experimental analyses of DPS. In this factorized scenario, one might notice that $\sigma_{D P S}$, i.e. the DPS cross section (see, e.g., Refs. $[2,41]$ ), depends on $1 / \bar{\sigma}_{\text {eff }}$ [41]. Thanks to this feature, the value of $1 / \bar{\sigma}_{\text {eff }}$ provides a rough estimate of the magnitude of $\sigma_{D P S}$. In the next section we will provide model predictions for hypotetical experiments with mesons.

\section{Calculation of the pion dPDF using an AdS/QCD model}

In the present section we introduce and discuss the LF wave function later used to evaluate the dPDFs. In particular, we will make use of the AdS/QCD soft-wall model approach. While in this framework several models exist [23,24,26,42], we have chosen the first one $[23,24]$, which we consider the most straightforward and therefore most suitable to show general properties of pion dPDFs. In this scheme, the pion w.f. reads:

$\psi_{\pi o}\left(x, \mathbf{k}_{\mathbf{1}}\right)=A_{o} \frac{4 \pi}{\kappa_{o} \sqrt{x(1-x)}} e^{-\frac{\mathbf{k}_{1 \perp}^{2}+m_{o}^{2}}{x(1-x) 2 \kappa_{o}^{2}}}$,

where $m_{o}=m_{u} \sim m_{\bar{d}}, x=x_{1}, x_{2}=1-x$ and $\mathbf{k}_{2} \perp=$ $-\mathbf{k}_{1} \perp$. The parameters of the model have been recently fixed to reproduce the Regge behavior of the mass spectrum of mesons [29,42]. They are $\kappa_{o}=0.523 \mathrm{GeV}$ and $m_{o} \sim 0.33$ $\mathrm{GeV}$. The constant $A_{o}$, is fixed by the normalization condition (4) and it is found to be $A_{o}=3.0498$. Using Eq. (17) in Eq. (7), the integrated $\mathrm{dPDF}$ is analytically expressed by:

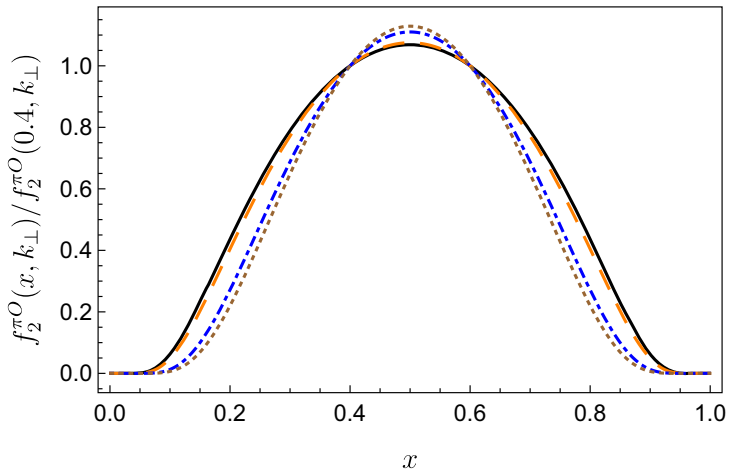

$\mathrm{GeV}$ and dotted line $k_{\perp}=0.6 \mathrm{GeV}$. Right panel: the ratio defined by Eq. (9) for the same parameters as in the left panel

$f_{2}^{\pi O}\left(x, \mathbf{k}_{\perp}\right)=A_{O}^{2} e^{-\frac{4 m_{D}^{2}+\mathbf{k}_{\perp}^{2}}{4 \kappa_{o}^{2} x(1-x)}}$.

For definiteness, we will consider in the following a $\pi^{+}$ system. The distributions for $\pi^{-}$and $\pi^{0}$ can be obtained by isospin and charge conjugation. As one can see in the left panel of Fig. 1, as it happens in the proton case $[9,10]$, the dPDF decreases as $k_{\perp}$ increases, and the factorization in $k_{\perp}$ and $x$ is not supported by the model as can be observed in the right panel of Fig. 1, where the the ratio (9) shows a clear $\mathbf{k}_{\perp}$ dependence. We conclude the discussion of these results by reporting the mean value of $\sigma_{\text {eff }}$ within the model at the hadronic scale $\mu_{0}, \bar{\sigma}_{e f f}^{\pi}\left(\mu_{0}\right)=41.69 \mathrm{mb}$. This value is larger than that corresponding to the proton case, i.e. $\bar{\sigma}_{\text {eff }} \sim 15$ $\mathrm{mb}[11,36,37]$. As demonstrated in Ref. [7], in general, the mean value of $\sigma_{e f f}$ is related to the geometrical structure of the colliding hadron, in particular to the transverse distance between two active partons in a DPS process. Such a relation provides a physical interpretation for the different values of $\bar{\sigma}_{e f f}$ found for the pion and for the proton. In particular, assuming $\bar{\sigma}_{\text {eff }}$ for the pion to be realistic, such a difference would suggest a different transverse distance between two partons in the proton and in the pion.

For completeness, we report in Fig. 2 the pion GPD evaluated within the model. As one can see, the pion GPD is very similar to the integrated $\mathrm{dPDF}$. It is apparent that the expressions for the dPDF and GPD, Eqs. (7, 13), in terms of the light-front pion wave function, are similar. However, in the integrated $\mathrm{dPDF}, k_{\perp}$ represents an intrinsic imbalance of the parton momentum between the initial and the final states keeping the same pion momentum in both states, while in the GPDs, $\Delta_{\perp}=k_{\perp}$ represents the difference in momentum between the initial and final state of the pion. Therefore, the dependence of the GPDs on the partonic momentum, i.e. $\mathbf{k}_{1, \perp} \pm(1-x) \mathbf{k}_{\perp}$ produces an asymmetry in the $x$ dependence, which is not present in dPDF. Moreover, since in the GPDs the momentum imbalance in the wave function is 


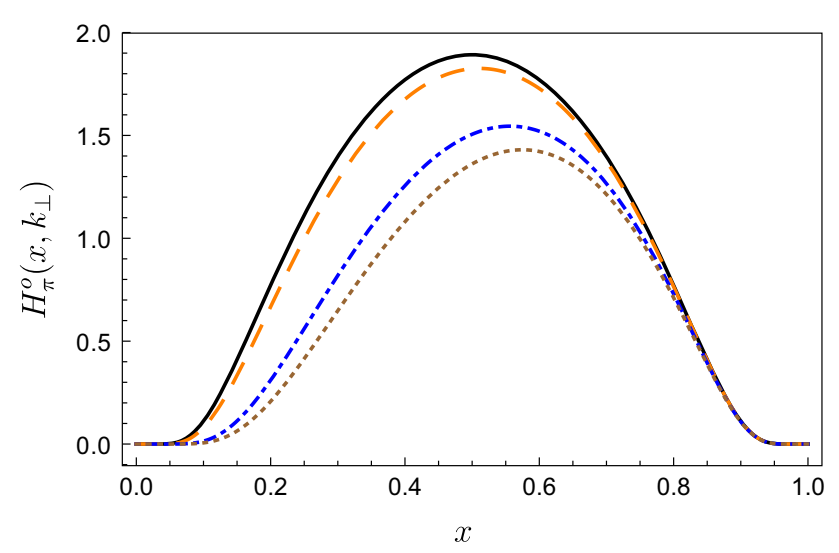

Fig. 2 The pion GPD defined in Eq. (13) for $k_{\perp}=0 \mathrm{GeV}$ (full line), $k_{\perp}=0.2 \mathrm{GeV}$ (dashed line), $k_{\perp}=0.5 \mathrm{GeV}$ (dot-dashed) line and $k_{\perp}=0.6 \mathrm{GeV}$ (dotted line)

multiplied by the pre-factor $1-x<1$ (see Eq. (13)), at variance with the $\mathrm{dPDF}$, the latter goes to zero faster then the GPD. Let us stress that such a similarity between dPDFs and GPDs holds only for the valence component and at the hadronic scale, i.e. where only two valence particles are taken into account in the model. If higher Fock states were included in the LF w.f. representation of the pion, other non perturbative $x_{1}-x_{2}$ correlations would appear. Moreover if one considers the $\mathrm{pQCD}$ evolution of dPDFs, also perturbative $x_{1}-x_{2}$ correlations show up (see e.g. Refs. [12,43]). Analogously to the proton case, this non trivial dependence of dPDFs on $x_{1}$ and $x_{2}$ cannot be accessed via GPDs, a confirmation of the rich three-dimensional structure accessible via dPDFs.

Finally we compare the complete $f_{2}^{\pi}\left(x, \mathbf{k}_{\perp}\right)$ with its approximation Eq. (12), i.e. $f_{2, A}^{\pi}\left(x, \mathbf{k}_{\perp}\right)$. If only the valence contribution were considered, the approximation to the integrated dPDF would become a product of a GPD and a form factor, as seen in Eq. (12), at variance with the proton case, where the dPDF is written as a product of two GPDs. In Fig. 3, we compare the integrated $\operatorname{dPDF}(7)$ and its approximation (12) as a function of $x$ for three different values of $k_{\perp}$. Similarly to what happens with the proton case [7$10,12,35,41,44]$, the approximation of one-body quantities gets worse and worse with increasing $k_{\perp}$. This fact points to non trivial information, contained in the pion $\mathrm{dPDF}$ at the hadronic scale, different from that encoded in the GPDs and ffs.

\section{Evolution}

The next step in our scheme is to calculate the perturbative evolution of the dPDFs from the low momentum scale of the model, the hadronic scale $\mu_{0}^{2}$, to the high scale of the data $Q^{2}$. As stated in the Sect. 1, dPDFs depend on two momentum scales. For simplicity, as it has been in done in

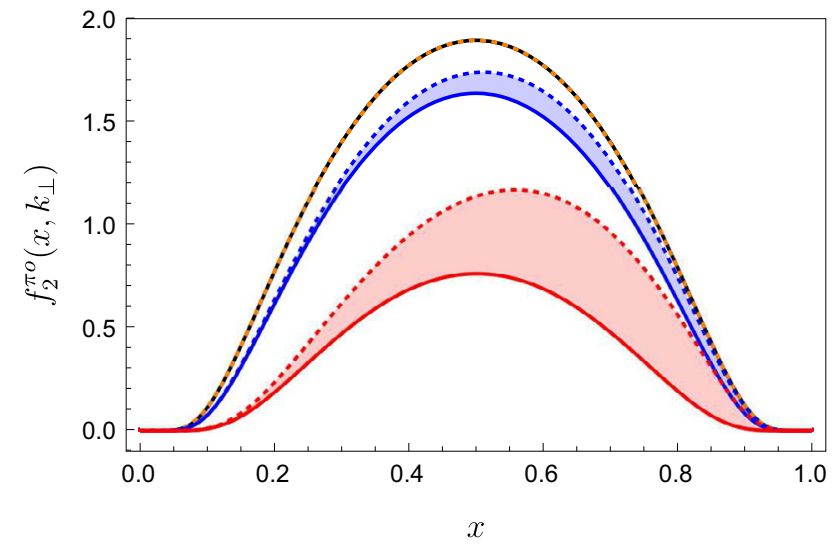

Fig. 3 The pion integrated dPDF, evaluated by means of its definition of Eq. (7), is shown in full lines, and its approximation, defined by Eq. (12), is plotted in dotted lines, for three values of $k_{\perp}: k_{\perp}=0 \mathrm{GeV}$, $k_{\perp}=0.2 \mathrm{GeV}$ and $k_{\perp}=0.5 \mathrm{GeV}$. The quality of the approximation decreases as $k_{\perp}$ increases as shown by the bands emphasizing the difference between the exact calculation and the approximation

previous works, see e.g. Ref. [45], we assume here that the two scales coincide. We follow here the same strategy developed in Refs. $[10,12]$ adapted to the use of quark models to calculate the proton's dPDFs. Historically, the evolution equations for dPDFs can be seen as a generalization of the usual DGLAP equations (see the original papers $[14,15]$ and recent contributions in Refs. [3,36,43,45-54]). This feature sets up the strategy which we are going to discuss next. Possible effects of the QCD evolution on the $\mathbf{k}_{\perp}$ dependence, presently under investigation and have not been considered here.

We start with the decomposition of the dPDF at a generic scale $Q^{2}$ :

$$
\begin{aligned}
F_{u \bar{d}} & =F_{\left(u_{V}+u_{s e a}\right)\left(\bar{d}_{V}+\bar{d}_{s e a}\right)}=F_{\left(u_{V}+\bar{u}\right)\left(\bar{d}_{V}+\bar{d}\right)} \\
& =F_{u_{V} \bar{d}_{V}}+F_{u_{V} \bar{d}}+F_{\bar{u} \bar{d}_{V}}+F_{\bar{u} \bar{d}}
\end{aligned}
$$

where, at the hadronic scale [55],

$u_{V}^{\pi^{+}}=\bar{d}_{V}^{\pi^{+}}=d_{V}^{\pi^{-}}=\bar{u}_{V}^{\pi^{-}}=v^{\pi} \equiv u_{V} ;$

while at any scale $q_{\text {sea }}=\bar{q}_{\text {sea }}=\bar{q}$, with $q=u, d, s$ for $N_{f}=3$ three active flavors. It is convenient to use the symmetrized form of dPDFs, $\bar{F}_{a b}=\left(F_{a b}+F_{b a}\right) / 2$ where $\bar{F}_{a b} \equiv \bar{F}_{a b}\left(x_{1}, x_{2}, \mathbf{k}_{\perp}, Q^{2}\right)$ is symmetric in $x_{1}, x_{2}$.

\subsection{Flavor decomposition}

In order to proceed with the evolution equations one has to construct from the $\bar{F}_{u \bar{d}}$ the Singlet and Non-Singlet components 
$\Sigma=\sum_{q} q^{+}=u_{V}+2 \bar{u}+d_{V}+2 \bar{d}+s+\bar{s}$

$T_{3}=u^{+}-d^{+}=u_{V}+2 \bar{u}-d_{V}-2 \bar{d}$

$T_{8}=u_{V}+2 \bar{u}+d_{V}+2 \bar{d}-2(s+\bar{s})$

$V_{i}=q_{i}^{-}$,

where $q_{i}^{ \pm}=q_{i} \pm \bar{q}_{i}$.

The evolution equations involve different equations for the Singlet-Singlet component $(\Sigma \Sigma)$, NonSinglet-Singlet components $\left(T_{8} \Sigma+\Sigma T_{8}, d_{V} \Sigma+\Sigma d_{V}, u_{V} \Sigma+\Sigma u_{V}\right)$, and NonSinglet - Non Singlet contributions (constructed from $\left.V_{i}, T_{3}, T_{8}\right)$.

\subsection{Mellin-moments and inversion}

The procedure follows by constructing the Mellin-moments which allow to solve the evolution equations easily. These quantities are

$$
\begin{aligned}
& \frac{1}{2} M_{\left(q_{a} q_{b}+q_{b} q_{a}\right)}^{n_{1} n_{2}}\left(Q^{2}\right)=\frac{M_{q_{b} q_{a}}^{n_{1} n_{2}}\left(Q^{2}\right)+M_{q_{a} q_{b}}^{n_{1} n_{2}}\left(Q^{2}\right)}{2} \\
& =\int_{0}^{1} d x_{1} \int_{0}^{1-x_{1}} d x_{2} x_{1}^{n_{1}-1} x_{2}^{n_{2}-1} \bar{F}_{a b}\left(x_{1}, x_{2} ; Q^{2}\right) .
\end{aligned}
$$

At the hadronic scale $\mu_{0}^{2}$, all the combinations of dPDFs with $\Sigma, T_{8}, T_{3}$ and $V_{i}$ will contain valence partons only. As a result the remaining term will be $F_{u_{V} \bar{d}_{V}}$, and the non vanishing moments at the hadronic scale will assume the form

$$
\begin{aligned}
& M_{u_{V} \bar{d}_{V}}^{n_{1} n_{2}}\left(\mu_{0}^{2}, \mathbf{k}_{\perp}\right) \\
& =\int_{0}^{1} d x_{1} \int_{0}^{1-x_{1}} d x_{2} \delta\left(1-x_{1}-x_{2}\right) \\
& \quad \times x_{1}^{n_{1}-1} x_{2}^{n_{2}-1} f_{2}^{\pi O}\left(x_{1}, x_{2}, \mathbf{k}_{\perp}, \mu_{0}^{2}\right) \\
& =\int_{0}^{1} d x x^{n_{1}-1}(1-x)^{n_{2}-1} f_{2}^{\pi O}\left(x, \mathbf{k}_{\perp}, \mu_{0}^{2}\right) .
\end{aligned}
$$

They enter the moments of the combinations directly depending on $\Sigma, T_{3}, T_{8}$ and $V_{i}$, but each moment $M_{a b}^{n_{1} n_{2}}\left(\mu_{0}^{2}\right)$, defined at the hadronic scale, will evolve according to its specific flavor symmetry [12]. The moments are independent functions of the complex indices $n_{1}, n_{2}$ and the inversion of Eq. (23) will produce dPDFs defined in the whole $\left(x_{1}, x_{2}\right)$ domain with $x_{1}+x_{2} \leq 1$.

\subsection{Evolution of the dPDFs: results}

In Fig. 4, we plot the second moment of the double distribution $\bar{F}_{u \bar{d}}\left(x_{1}, x_{2}, y, Q^{2}\right)$ defined by

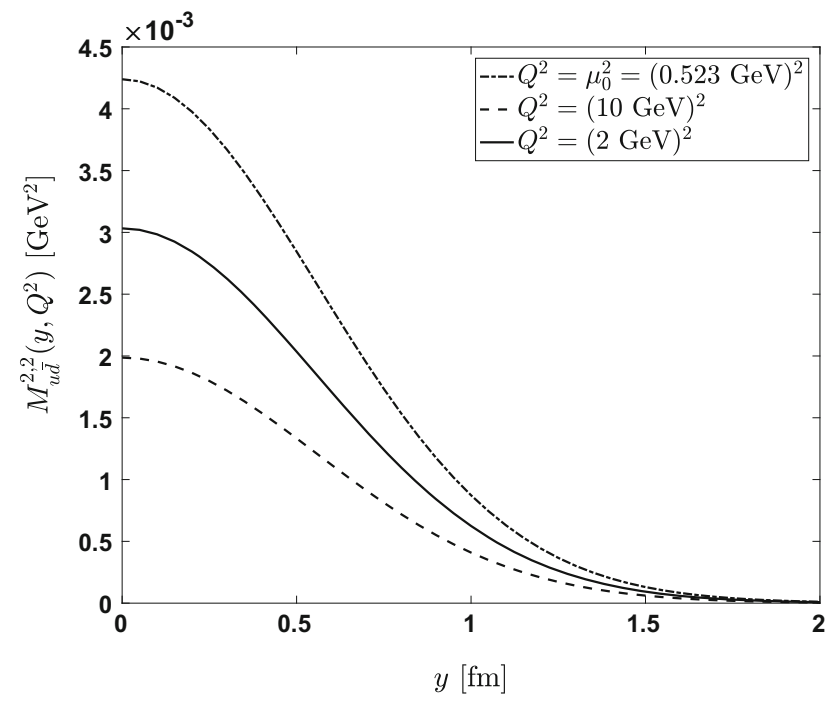

Fig. 4 The second moment of dPDFs, Eq. (24) as a function of the distance $y$ and at different scales: the hadronic scale $\mu_{0}^{2}=(0.523 \mathrm{GeV})^{2}$, $Q^{2}=4 \mathrm{GeV}^{2}$ and $100 \mathrm{GeV}^{2}$

$$
\begin{aligned}
& M_{u \bar{d}}^{22}\left(y, Q^{2}\right) \\
& \quad=\int_{0}^{1} d x_{1} \int_{0}^{1-x_{1}} d x_{2} x_{1} x_{2} \bar{F}_{u \bar{d}}\left(x_{1}, x_{2}, y, Q^{2}\right),
\end{aligned}
$$

where $y$ is the distance between the two correlated partons, obtained Fourier transforming the $\mathbf{k}_{\perp}$ dependent distribution $f_{2}^{\pi O}\left(x, \mathbf{k}_{\perp}\right)$ given in Eq. (18). This quantity incorporates the evolution to large $Q^{2}$ of the distribution $\bar{F}_{u \bar{d}}\left(x_{1}, x_{2}, y, Q^{2}\right)$ starting from the initial scale $\mu_{0}^{2}=(0.523)^{2} \mathrm{GeV}^{2}$, already used in calculation of pion PDF and unpolarized transverse momentum dependent PDF in Ref. [30].

At the hadronic scale since only two valence particles are present, the support condition, preserved within the lightfront approach, forces the dPDF to exist only when $x_{1}+$ $x_{2}=1$. This is at variance with the proton case where the existence of a third particle allows complete freedom for $x_{1}$ and $x_{2}$ as long as momentum is conserved $x_{1}+x_{2}<1$. The evolution procedure, described by Eq. (22), where $n_{1}$ and $n_{2}$ are independent complex parameters allows to obtain $F_{u \bar{d}}\left(x_{1}, x_{2}, y, Q^{2}\right)$ for all values of $x_{1}$ and $x_{2}$ and $x_{1}+x_{2}<$ 1. The creation, in the evolution process, of sea and glue partons allows $x_{1}$ and $x_{2}$ to free themselves from the valence condition.

As we mentioned in the Sect. 1, preliminary results for quantities related to moments of the pion dPDFs have been recently reported within a lattice $\mathrm{QCD}$ approach [31]. A comparison of results obtained in model calculations with lattice data would open interesting new perspectives.

A first important effect of the evolution procedure can be seen in Fig. 5, where the double distribution $x_{1} x_{2} \bar{F}_{u \bar{d}}\left(x_{1}, x_{2}\right.$, $\left.y, Q^{2}=100 \mathrm{GeV}^{2}\right)$ is shown in the domain $\left(x_{1}, x_{2}=1-x_{1}\right)$ as a function of $x_{1}$ and for different values of $y$. The compar- 


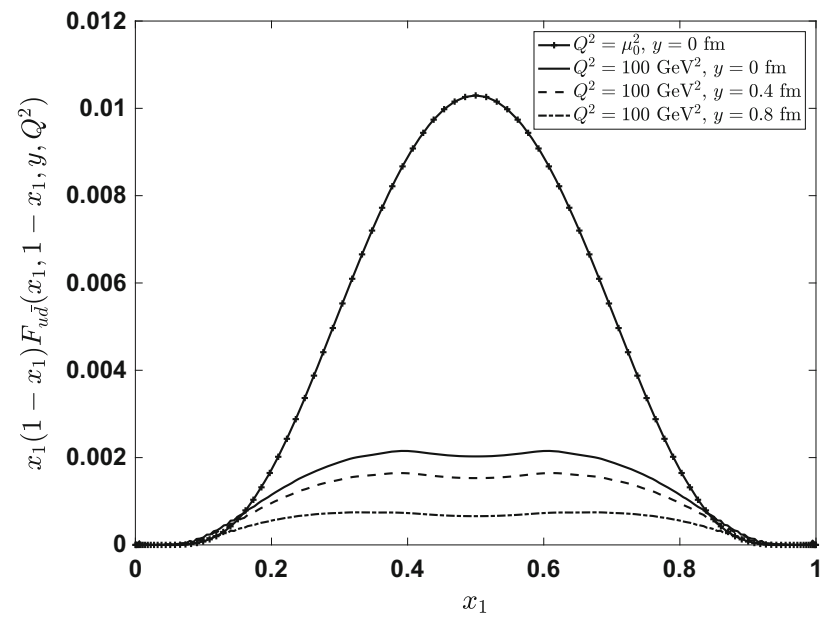

Fig. 5 The quantity $x_{1}\left(1-x_{1}\right) F_{u \bar{d}}\left(x_{1}, 1-x_{1}, y, Q^{2}=100 \mathrm{GeV}^{2}\right)$ is plotted as a function of $x_{1}$ at different $y$-values. The input distribution at $\mu_{0}^{2}$ and $y=0 \mathrm{fm}$ is also shown

ison with the same distribution at the hadronic scale $\mu_{0}^{2}$ and $y=0$ clearly emphasizes the effects of the evolution. The evolution from $\mu_{0}^{2}$ to $Q^{2}=100 \mathrm{GeV}^{2}$ produces a reduction of the distribution, a behavior physically interpretable as the creation of new partonic species carrying momentum, in particular gluon distributions. Recall that the latter are zero at the hadronic scale for the models considered. In Fig. 6 the double distribution $x_{1} x_{2} \bar{F}_{u_{V} g}\left(x_{1}, x_{2}, y, Q^{2}\right)$ is plotted. The upper panel shows the dependence of the distribution on the scale $Q^{2}$, while the lower panel illustrates its dependence on the parton distance $y$.

A large part of the valence parton momentum is transferred to the gluons which increases dramatically at low- $x$, while the relevance of the valence partons decreases. In closing this section we show, in Fig. 7, how the approximation Eq. (12) is violated also at the high momentum scale, i.e. $Q^{2}=4 \mathrm{GeV}^{2}$. As one can observe, the amount of the violation of the ansatz Eq. (12), at both the initial hadronic scale and the final one, is essentially the same. Such a feature relies on the properties of the pQCD evolution equation of standard PDFs. In fact, being $f_{2}^{\pi}\left(x, k_{\perp}\right)$ the integral over $x_{2}$ of the dPDF, it evolves as an usual PDF [4] and as the GPDs for zero skewness. Since this kind of procedure preserves the $k_{\perp}$ dependence of the distributions, all correlations between $x$ and $k_{\perp}$ survive also at high momentum scales. We reiterate that here only the $Q^{2}$ evolution of the $x$ dependence is performed.

\section{Conclusions}

Double parton distribution functions may represent a novel tool to access the three dimensional structure of hadrons. It is therefore natural to study the dPDFs of the pions, specially now that the first estimates of quantities related to the dPDF of
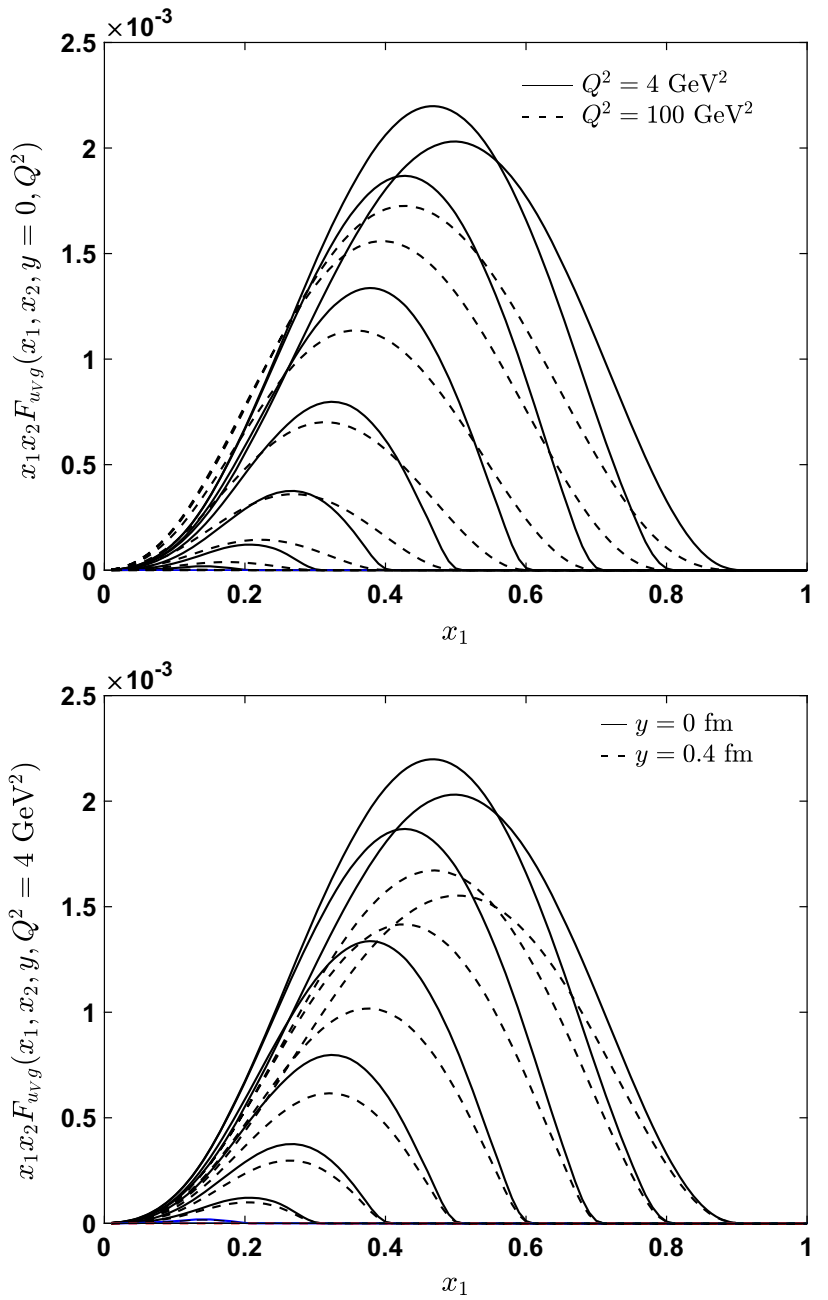

Fig. 6 The quantity $x_{1} x_{2} F_{u_{V} g}\left(x_{1}, x_{2}, y, Q^{2}\right)$ is plotted as a function of $x_{1}$ for $x_{2}=0.1,0.2,0.3,0.4,0.5,0.6,0.7,0.8,0.9$. In the upper panel the full lines represent the results at $Q^{2}=4 \mathrm{GeV}^{2}$ and the dashed lines those ones at $Q^{2}=100 \mathrm{GeV}^{2}$ for the same value of the parton distance $y=0 \mathrm{fm}$. The lower panel compares the distributions for different distances: $y=0 \mathrm{fm}$ (full lines) and $y=0.4 \mathrm{fm}$ (dashed lines) and $Q^{2}=4 \mathrm{GeV}^{2}$

pion have been reported by lattice studies [31]. We have used here a light front formalism, for which the wave function of the system is required. The AdS/QCD correspondence has generated our LF wave function. Once the formalism has been set up we have calculated several quantities Among them, we have obtained the mean effective cross section for pion-pion scattering at the hadronic scale, $\bar{\sigma}_{\text {eff }}^{\pi}\left(\mu_{0}\right)=41$ $\mathrm{mb}$, which turns out to be larger than the same cross section, evaluated with a similar approach, in the proton case. This quantity is very much independent on QCD evolution and provides us with an estimate of the magnitude of DPS [11, 13].

In the adopted AdS/QCD model, dPDFs turn out to be analytical. It has been found that an approximation in terms of generalized parton distributions, proposed in several 


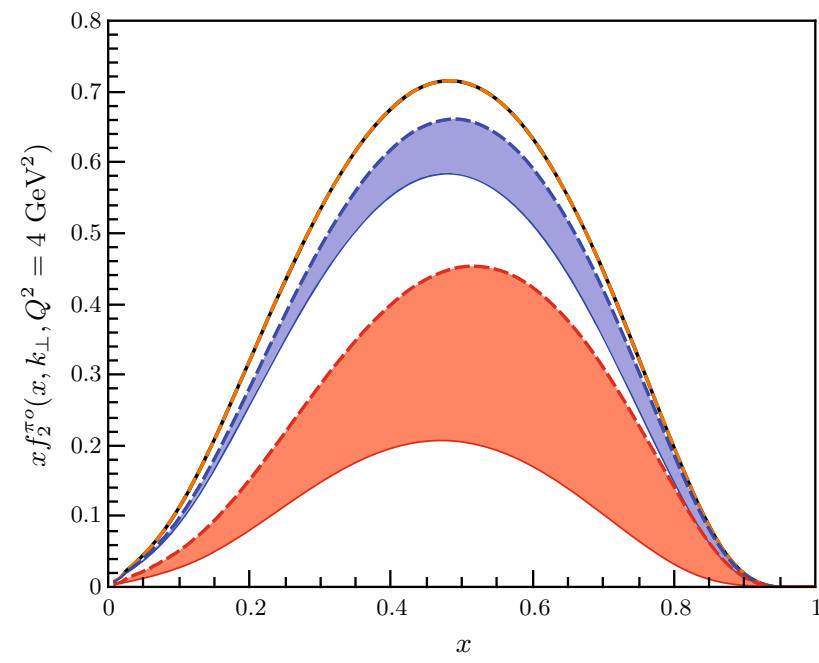

Fig. 7 The quantity $x f_{2}^{\pi o}\left(x_{1}, k_{\perp}, Q^{2}=4 \mathrm{GeV}^{2}\right)$, full lines, compared with its approximation, Eq. (12), dashed lines, for three values of $k_{\perp}$ : $k_{\perp}=0 \mathrm{GeV}, k_{\perp}=0.2 \mathrm{GeV}$ and $k_{\perp}=0.5 \mathrm{GeV}$. The bands illustrate the difference between the exact calculation and the approximation

approaches, is not reliable, as it happens also in the proton case [12]. Analogously, our calculations show that dPDFs do not factorize into $x_{1,2}$ - and $k_{\perp}$-dependent terms. These facts expose the presence of unknown double parton correlations in the pion not accessible from one-body distributions.

We have performed the evolution to high $Q^{2}$ using the conventional formalism, subject in this case, at the model momentum scale where only two valence constituents with momentum fractions $x_{1}$ and $x_{2}$ are present, to the $x_{1}+x_{2}=1$ restriction. Expected results are obtained. For example, the second moment decreases as $Q^{2}$ increases, signalling the opening of new dPDFs associated with sea and gluons. A good example has been shown in Fig. 6, where the dPDF due to the correlation of valence and gluons is shown for different values of the parton distance and $Q^{2}$. At the hadronic scale such a distribution vanishes because no gluons are included. At higher scale $Q^{2}$ the radiative production of gluons from the valence system makes $F_{u_{V} g}>0$. The dPDFs show, both at the model scale and at a high momentum scale, also a strong dependence on the partonic distance, decreasing in magnitude as the distance increases. While, at present, experiments designed to measure dPDFs of the pion cannot be imagined, lattice calculations have started to approach this problem and will be likely able, in the near future, to distinguish between predictions of different models of the pion structure, such as the one presented here, and addressing possible effects of spin correlations, opening new perspectives.

Acknowledgements The present study started thanks to a discussion with Daniele Treleani. This work was supported in part by the Mineco under contract FPA2013-47443-C2-1-P and SEV-2014-0398. M. T. and V. V. thank the University of Perugia and INFN, Perugia section, for warm hospitality and support. S. S. thanks the University of Valencia and the IFIC for warm hospitality and support.

Open Access This article is distributed under the terms of the Creative Commons Attribution 4.0 International License (http://creativecomm ons.org/licenses/by/4.0/), which permits unrestricted use, distribution, and reproduction in any medium, provided you give appropriate credit to the original author(s) and the source, provide a link to the Creative Commons license, and indicate if changes were made.

Funded by SCOAP ${ }^{3}$.

\section{References}

1. G. Aad et al., ATLAS Collaboration, New J. Phys. 15, 033038 (2013)

2. N. Paver, D. Treleani, Nuovo Cim. A 70, 215 (1982)

3. M. Diehl, D. Ostermeier, A. Schafer, JHEP 03, 089 (2012)

4. J.R. Gaunt, W.J. Stirling, JHEP 1003, 005 (2010)

5. M. Guidal, H. Moutarde, M. Vanderhaeghen, Rep. Prog. Phys. 76, 066202 (2013)

6. R. Dupré, M. Guidal, M. Vanderhaeghen, Phys. Rev. D 95(1), 011501 (2017)

7. M. Rinaldi, F.A. Ceccopieri, Phys. Rev. D 97(7), 071501 (2018)

8. H.M. Chang, A.V. Manohar, W.J. Waalewijn, Phys. Rev. D 87(3), 034009 (2013)

9. M. Rinaldi, S. Scopetta, V. Vento, Phys. Rev. D 87, 114021 (2013)

10. M. Rinaldi, S. Scopetta, M. Traini, V. Vento, JHEP 12, 028 (2014)

11. M. Rinaldi, S. Scopetta, M. Traini, V. Vento, Phys. Lett. B 752, 40 (2016)

12. M. Rinaldi, S. Scopetta, M.C. Traini, V. Vento, JHEP 16, 063 (2016)

13. M. Traini, M. Rinaldi, S. Scopetta, V. Vento, Phys. Lett. B 768, 270 (2017)

14. R. Kirschner, Phys. Lett. B 84, 266 (1979)

15. V.P. Shelest, A.M. Snigirev, G.M. Zinovev, Phys. Lett. B 113, 325 (1982)

16. J.M. Maldacena, Int. J. Theor. Phys. 38, 1113 (1999)

17. J.M. Maldacena, Adv. Theor. Math. Phys. 2, 231 (1998)

18. E. Witten, Adv. Theor. Math. Phys. 2, 253 (1998)

19. J. Polchinski, M.J. Strassler, arXiv:hep-th/0003136

20. S.J. Brodsky, G.F. de Teramond, Phys. Lett. B 582, 211 (2004)

21. J. Erlich, E. Katz, D.T. Son, M.A. Stephanov, Phys. Rev. Lett. 95, $261602(2005)$

22. L. Da Rold, A. Pomarol, Nucl. Phys. B 721, 79 (2005)

23. S.J. Brodsky, G.F. de Teramond, Phys. Rev. D 77, 056007 (2008)

24. S.J. Brodsky, G.F. de Teramond, Subnucl. Ser. 45, 139 (2009)

25. J.R. Forshaw, R. Sandapen, Phys. Rev. Lett. 109, 081601 (2012)

26. G.F. de Teramond et al., HLFHS Collaboration, Phys. Rev. Lett. 120(18), 182001 (2018)

27. M.C. Traini, Eur. Phys. J. C 77(4), 246 (2017)

28. M. Rinaldi, Phys. Lett. B 771, 563 (2017)

29. R. Swarnkar, D. Chakrabarti, Phys. Rev. D 92(7), 074023 (2015)

30. A. Bacchetta, S. Cotogno, B. Pasquini, Phys. Lett. B 771, 546 (2017)

31. C. Zimmermann, RQCD Collaboration, PoS LATTICE 2016, 152 (2016)

32. T. Kasemets, A. Mukherjee, Phys. Rev. D 94(7), 074029 (2016)

33. S.J. Brodsky, H.C. Pauli, S.S. Pinsky, Phys. Rep. 301, 299 (1998)

34. S.J. Brodsky, M. Diehl, D.S. Hwang, Nucl. Phys. B 596, 99 (2001)

35. M. Rinaldi, F.A. Ceccopieri, Phys. Rev. D 95(3), 034040 (2017)

36. B. Blok, Y. Dokshitser, L. Frankfurt, M. Strikman, Eur. Phys. J. C 72, 1963 (2012)

37. B. Blok, Y. Dokshitzer, L. Frankfurt, M. Strikman, Eur. Phys. J. C 74, 2926 (2014)

38. M. Diehl, Phys. Rep. 388, 41 (2003) 
39. C. Mezrag, L. Chang, H. Moutarde, C.D. Roberts, J. RodríguezQuintero, F. Sabatié, S.M. Schmidt, Phys. Lett. B 741, 190 (2015)

40. A. Del Fabbro, D. Treleani, Phys. Rev. D 63, 057901 (2001)

41. F.A. Ceccopieri, M. Rinaldi, S. Scopetta, Phys. Rev. D 95(11), 114030 (2017)

42. M. Ahmady, F. Chishtie, R. Sandapen, Phys. Rev. D 95(7), 074008 (2017)

43. M. Diehl, T. Kasemets, S. Keane, JHEP 05, 118 (2014)

44. T. Kasemets, S. Scopetta, arXiv:1712.02884 [hep-ph]

45. A.M. Snigirev, N.A. Snigireva, G.M. Zinovjev, Phys. Rev. D 90(1), 014015 (2014)
46. A.V. Manohar, W.J. Waalewijn, Phys. Rev. D 85, 114009 (2012)

47. E. Cattaruzza, A. Del Fabbro, D. Treleani, Phys. Rev. D 72, 034022 (2005)

48. M. Diehl, A. Schafer, Phys. Lett. B 698, 389 (2011)

49. M.G. Ryskin, A.M. Snigirev, Phys. Rev. D 83, 114047 (2011)

50. J.R. Gaunt, W.J. Stirling, JHEP 06, 048 (2011)

51. F.A. Ceccopieri, Phys. Lett. B 697, 482 (2011)

52. J.R. Gaunt, JHEP 01, 042 (2013)

53. A.V. Manohar, W.J. Waalewijn, Phys. Lett. B 713, 196 (2012)

54. W. Broniowski, E. Ruiz Arriola, Few Body Syst. 55, 381 (2014)

55. M. Gluck, E. Reya, M. Stratmann, Eur. Phys. J. C 2, 159 (1998) 